\title{
Design and physicochemical stability studies of paediatric oral formulations of sildenafil
}

\author{
${ }_{4}$ Q1 N. Provenza ${ }^{a}$, A.C. Calpena ${ }^{a}$, M. Mallandrich ${ }^{a}$, L. Halbaut ${ }^{a}$, B. Clares ${ }^{b}, *$ \\ a Department of Biopharmacy and Pharmaceutical Technology, School of Pharmacy, University of Barcelona, Av. Joan XXIII s/n, 08028 Barcelona, Spain \\ ${ }^{\mathrm{b}}$ Department of Pharmaceutical Technology, School of Pharmacy, University of Granada, Campus of Cartuja street s/n, 18071 Granada, Spain
}

\section{A R T I C L E I N F O}

\section{Article history:}

Received 31 August 2013

Accepted 3 November 2013

Available online $\mathrm{xxx}$

\section{Keywords:}

Sildenafil oral formulations

Paediatric

Stability

Drug administration

\begin{abstract}
A B S T R A C T
Personalized medicine is a challenging research area in paediatric treatments. Elaborating new paediatric formulations when no commercial forms are available is a common practice in pharmacy laboratories; among these, oral liquid formulations are the most common. But due to the lack of specialized equipment, frequently studies to assure the efficiency and safety of the final medicine cannot be carried out. Thus the purpose of this work was the development, characterization and stability evaluation of two oral formulations of sildenafil for the treatment of neonatal persistent pulmonary hypertension.

After the establishment of a standard operating procedure (SOP) and elaboration, the physicochemical stability parameters appearance, $\mathrm{pH}$, particle size, rheological behaviour and drug content of formulations were evaluated at three different temperatures for 90 days. Equally, prediction of long term stability, as well as, microbiological stability was performed. Formulations resulted in a suspension and a solution slightly coloured exhibiting fruity odour. Formulation I (suspension) exhibited the best physicochemical properties including Newtonian behaviour and uniformity of API content above $90 \%$ to assure an exact dosification process.
\end{abstract}

(c) 2013 Published by Elsevier B.V.

\section{Introduction}

Sildenafil (SLD) (1-[4-ethoxy-3-(6,7-dihydro-1-methyl-7-oxo3-propyl-1H-pyrazolo[4,3-d] pyrimidin-5-yl) phenylsulphonyl]-4methyl piperazine (Fig. 1) is a selective phosphodiesterase inhibitor type 5 (PDE5) that reduces pulmonary vascular resistance (Sola and Baquero, 2007) producing vasodilatation by increasing cyclic guanosine monophosphate an intracellular second messenger that has been implicated in maintaining the low tone of the normal pulmonary vascular bed (Gold et al., 1990).

The use of SLD as a pulmonary vasodilator in infant with congenital heart disease was first reported in a small case series post-operatively in 1999 (Atz and Wessel, 1999). Currently, SLD is used frequently for long-term treatment of children with pulmonary arterial hypertension (Humpl et al., 2005). The incidence of neonatal persistent pulmonary hypertension (PPHN) in term or near-term infants is reported to vary between 0.43 and 6.8 of 1000 live births and mortality remains at $10-20 \%$ (Travadi and Patole, 2003). It is characterized by a dysbalance between vasodilatation and vasoconstriction, in which vasoconstriction prevails resulting

\footnotetext{
* Corresponding author at: Department of Pharmacy and Pharmaceutical Technology, Faculty of Pharmacy, University of Granada, Campus of Cartuja street s/n, 18071 Granada, Spain. Tel.: +34 958 243904; fax: +34 958248958.

E-mail address: beatrizclares@ugr.es (B. Clares).
}

in severe pulmonary vascular resistance (Steinhorn et al., 2009). Most prominent histological changes in PPHN include hypertrophy of the perivascular muscular layer in small and large pulmonary arteries (Hoehn, 2007).

SLD was first commercially available as Viagra ${ }^{\circledR}$ for the treatment of male erectile dysfunction. Under the trade name of Revatio ${ }^{\circledR}$, it has recently been used for treatment of pulmonary hypertension in adults (Archer and Michelakis, 2009). In this context, personalized medicine is a current and challenging research area because paediatrics are more vulnerable to drug administration errors due to a lack of appropriate drug dosages and strengths for use in this group of patients (Bauters et al., 2012) for this, both community and hospital pharmacists are often challenged with the preparation of a dosage form not commercially available using traditional pharmaceutical compounding techniques as an alternative (Glass and Haywood, 2006). On the other hand the homogeneous distribution of the pure drug is an additional problem if it is formulated in low proportion (Sundell-Bredenberg and Nyströn, 2001).

Up to $37 \%$ of the drugs used in community practice settings and up to $80 \%$ of the drugs used in neonatal intensive care, are prescribed in an off-label or unlicensed manner (Wilson et al., 1998). In addition the use of the active pharmaceutical ingredient (API) by modifying commercially available tablets or capsules is not allowed by legislation in some countries (Santoveña et al., 2012). For this reason, in the design and formulation of dosage forms the physical, chemical and biological properties of the active substance, as well 


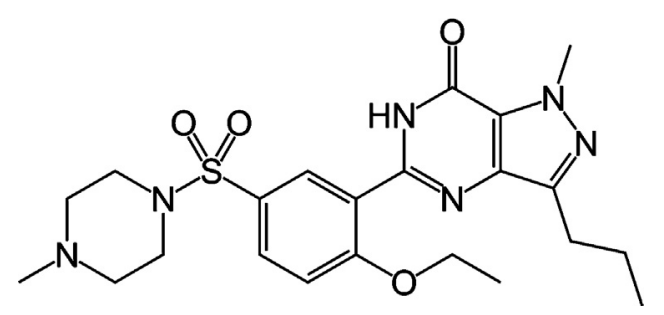

Fig. 1. Chemical structure of sildenafil.

as, the rest of pharmaceutical ingredients used should be seriously considered (Nahata and Allen, 2008).

Orally administered liquids are still considered the standard forms for these patients (Bauters et al., 2012), because a single liquid paediatric preparation may be used for infants and children of all ages, with the dose of the drug varied by the volume administered (Allen, 2008) reducing potential dosage mistakes, and helping the treatment adherence. The absence of suitable medicines, critical safety or efficacy information bears various risks as continuously claimed by different organizations particularly with this vulnerable patient population (Ernest et al., 2007; Wening and Breitkreutz, 2011).

Most common types of drug administration errors are incorrect time of administration (28.8\%), followed by incorrect drug preparation (26\%), omission errors (16.3\%) and incorrect dose (11.5\%), and many studies have reported the dosing errors as the most common (Chua et al., 2010). However, a major problem remains with many liquid preparations due to the absence of information regarding suitability and stability (Brion et al., 2003). The variability in the design of such preparations, sometimes with an unknown or low stability, leads to the possibility of medication errors when handling dosages. On the other hand, no dose or stability control is performed in community or hospital pharmacies for every formulation prepared (Santoveña et al., 2012). For this reason, there are no studies either about the quality of the final product or about the dose homogeneity to be administered.

Therefore, the purpose of the present work was the design of paediatric oral formulations of SLD and the development of a simple and feasible SOP for its use by pharmacist both in community and hospital facilities. The physicochemical and microbiological stability of two different formulations $(2 \mathrm{mg} / \mathrm{mL}$ ) of SLD (pure powder) were evaluated to guarantee the correct dose administration, the efficiency of the treatment and the formulation stability during its preparation and storing.

\section{Materials and methods}

SLD citrate and excipients were pharmacopoeia grade and were provided by Acofarma S.A. (Barcelona, Spain). All other chemicals were all of analytical grade (Sigma-Aldrich, Spain). Double distilled water was used after filtration in a Milli- $Q^{\circledR}$ Gradinet A10 system apparatus (Millipore Iberica S.A.U.; Madrid, Spain).

\subsection{General SOP}

Compositions of the developed formulations of SLD are reported in Table 1 and were elaborated according to the following SOP: SLD is accurately weighed and added to the right amount of buffer citrate solution and water, and then the mixture is sonicated for $5 \mathrm{~min}$. Subsequently, the vehicle (simple syrup for formulation I and simple syrup glucose free for formulation II) are slowly added. Finally, mixtures are completed with water until reaching the volume and newly sonicated until homogeneity.
Table 1

Formulations composition.

\begin{tabular}{lll}
\hline Ingredients & Formulation 1 & Formulation 2 \\
\hline Sildenafil citrate $(\mathrm{mg})$ & 200 & 200 \\
Citrate buffered solution 0.1 $\mathrm{M}(\mathrm{pH}=4)(\mathrm{mL})$ & 10 & 10 \\
Excipient for syrup $(\mathrm{mL})$ & 45 & - \\
Excipient for syrup sugar free $(\mathrm{mL})$ & - & 45 \\
Bidistilled water $(\mathrm{mL})$ & q.s. 100 & q.s. 100 \\
\hline
\end{tabular}

As controls, the same amounts of formulations without drug (blank samples) were similarly prepared.

\subsection{Physicochemical characterization of formulations}

Aliquots of $10 \mathrm{~mL}$ of the suspensions were stored in amber glass containers at three different temperatures $\left(4,25\right.$ and $\left.40^{\circ} \mathrm{C}\right)$ for 90 days. Measures were performed at pre-selected times and comprised physicochemical testing of quantifiable parameters which could possibly change during storage period, such as appearance, $\mathrm{pH}$, particle size, rheological behaviour, and drug concentration. Preparations are considered stable if physical characteristics have not changed and drug concentration has remained above $90 \%$ of the original concentration.

\subsubsection{Appearance}

The physical appearance properties were studied using a visual examination method of the samples stored at each temperature, thus parameters as odour, colour or tendency to spontaneously form precipitates could be appreciated.

\subsection{2. $\mathrm{pH}$ measurements}

$\mathrm{pH}$ values were measured in triplicate using a digital $\mathrm{pH} / \mathrm{mV}$ meter micro-pH 200 (Crison Instruments S.A., Barcelona, Spain). A significant variance of $\mathrm{pH}$ over an adequate value per each formula could indicate a degradation of the pharmaceutical compounding or an erroneous elaboration. Particle size

Particle size analysis of formulation 1 was carried out by laser diffractometry (LD) using a Malvern Mastersizer 2000E (Malvern Instruments, Malvern, UK) yielding the volume distribution of the particles. The average particle size distribution was measured from 3 replicates of each sample. For the LD analysis the diameters 10\%, $50 \%$ and $90 \%$ were used. Measurements of the samples stored $25^{\circ} \mathrm{C}$ were performed at 0 and 90 days.

\subsubsection{Rheological behaviour}

The rheological characterization of the formulations was performed at $25^{\circ} \mathrm{C}$ using a rotational rheometer HAAKE Rheostress 1 (Thermo Fisher Scientific, Karlsruhe, Germany) equipped with a parallel plate geometry set-up with a fixed lower plate and an upper plate (Haake PP60 Ti, $6 \mathrm{~cm}$ diameter). Different gaps between plates were tested and a separation of $0.5 \mathrm{~mm}$ was selected. The rheometer was connected to a computer provided with the software HAAKE RheoWin ${ }^{\circledR}$ Job Manager V. 3.3 to carry out the test and RheoWin ${ }^{\circledR}$ Data Manager V 3.3 (Thermo Electron Corporation, Karlsruhe, Germany) to carry out the analysis of the obtained data. Viscosity curves and flow curves were recorded for 3 min during the ramp-up period from 0 to $100 \mathrm{~s}^{-1}, 1 \mathrm{~min}$ at $100 \mathrm{~s}^{-1}$ (constant share rate period) and finally 3 min during the ramp-down period from 100 to $0 \mathrm{~s}^{-1}$. Viscosity values at $100 \mathrm{~s}^{-1}$ were determined at $t_{0}$ and $t=90$ days for the samples stored at 4,25 and $40^{\circ} \mathrm{C}$ in triplicate.

\subsubsection{Dose content and quantification method}

The API content in samples was calculated in triplicate using the methodology described below. For this, $1 \mathrm{~mL}$ of sample (equivalent to $2 \mathrm{mg}$ of SLD) was added to $100 \mathrm{~mL}$ in glass amber volumetric flask 
obtaining a final SLD concentration of $20 \mu \mathrm{g} / \mathrm{mL}$. This solution was sonicated in an ultrasonic bath p-selecta 514 (Vidrafoc, Barcelona, Spain) for $1 \mathrm{~min}$.

Absorbance of the samples was conducted by an Amersham Biosciences Ultrospec 1100 Pro UV/Visible spectrophotometer (Amersham Biosciences, Piscataway, NJ, USA) at room temperature in $1 \mathrm{~cm}$ quartz cells at $292.5 \mathrm{~nm}$.

Working standard solutions for the calibration curves were prepared daily as follows; $25 \mathrm{mg}$ of SLD was accurately weighed and transferred to a $25 \mathrm{~mL}$ volumetric flask. Then it was dissolved in glacial acetic acid $0.1 \mathrm{M}$ to obtain a final SLD concentration of $1000 \mu \mathrm{g} / \mathrm{mL}$. From this solution, six standard stocks were prepared.

Validation of the developed methodology was carried out as per the international conference on harmonization guidelines Q2 (R1) (ICH, 2006) and included an evaluation of the following characteristics: linearity, sensitivity, accuracy and precision.

The linearity of the present method was evaluated by constructing the calibration curve at six concentration levels from 50 to $0.87 \mu \mathrm{g} / \mathrm{mL}$. The calibration curve was validated interday $(n=6)$ by different analysts and developed by plotting the instrument measurements versus the corresponding drug concentration. The least squares fit method was employed to statistically evaluate the results for linearity by a regression line and the corresponding slope, $y$-intercept and coefficient of linear correlation $\left(r^{2}\right)$. Furthermore, linearity was determined by one-way analysis of variance (ANOVA) test to compare the absorbances versus nominal concentrations of each standard, and differences were considered statistically significant when $p<0.05$. The least square linear regression analysis and mathematical determinations were performed by the Prism ${ }^{\circledR}$, V. 3.00 software (GraphPad Software Inc., San Diego, CA, USA).

The selectivity of different assays was confirmed by the individual analysis of blank samples assuring that no interferences took place.

Sensitivity of the method was determined with respect to limit of detection (LOD) and limit of quantification (LOQ). LOD provides information on minimum level at which the SLD was reliably detected by the analysis of samples with known concentrations of SLD and LOQ in the same way, establishes the minimum level at which SLD was quantified with acceptable accuracy and precision. Both parameters, LOD and LOQ were determined based on the standard deviation of the response and the slope of the calibration curve using the formula:

$\mathrm{LOD}$ or $\mathrm{LOQ}=k \times \frac{\mathrm{SD}_{\mathrm{Sa}}}{\mathrm{Sb}}$

where $k$ is the a factor related to the level of confidence, its value is 3.3 for LOD and 10 for LOQ. SD $\mathrm{Sa}$ is the standard deviation of the intercept and Sb is the slope (McEvoy et al., 2007).

Accuracy was defined as the percentage of the systematic error, which is determined as standardized agreement between the measured value and the true value (relative error $<10 \%$ ). Finally, the precision of the assay was determined by the intraday repeatability, for this, the relative standard deviation (RSD) of replicates analyzed during the same day (while keeping the operating conditions identical) was calculated. Similarly, the intermediate precision (interday) was assessed by repeated analysis of the control samples in six different days.

\subsubsection{Stability studies}

Homogeneity of the formulations was examined over time $(0$, $7,15,30,60$ and 90 days) at three temperatures to ensure that every vial contained no less than $90 \%$ and no more than $110 \%$ of the theoretically calculated and labelled amount of SLD per unit of volume (The United States Pharmacopoeia, 2007). Six samples were taken from each vial and SLD content was calculated using the methodology described below. The relative standard deviation (\%RSD) was used to assess the homogeneity of the suspensions.

\subsection{Optical stability study}

In order to predict the long term stability of formulation 1 , Measurements of suspensions by multiple light scattering were performed in triplicate using the Turbiscan ${ }^{\circledR}$ Lab (Formulaction Co., L'Union, France), an optical instrument that characterizes concentrated dispersions. The analyzed suspension is placed on a cylindrical glass measuring cell. The light source is a pulsed near infrared $(\lambda=880 \mathrm{~nm})$. Undiluted samples $(35 \mathrm{~mL})$ were placed and kept on a cylindrical glass measuring cell which was completely scanned by a reading head. A pattern of the light flux as a function of the sample height was obtained, giving a macroscopic fingerprint of the sample at a given time. Measurements were performed at room temperature.

\subsection{Microbiological studies}

Microbiological tests of suspensions were performed at 0 and 90 days according to the European Pharmacopoeia monograph of nonsterile products (European Pharmacopoeia, 2013). The microbial count was considered to be the average number of colony forming units (cfu) found in agar. Liquid oral formulations would meet microbial requirements if the total aerobic microbial count were less than $10^{2} \mathrm{cfu} / \mathrm{mL}$, the total combined yeast/mould count less than $10^{1} \mathrm{cfu} / \mathrm{mL}$ and confirmed the absence of Escherichia coli.

\subsection{Statistical analysis}

Data were statistically analyzed by one-way ANOVA, followed by $t$-student test and represented as the mean of $n$ replicates $\pm S D$. The level of significance was set at $p<0.05$ using Prism ${ }^{\circledR}$, V. 3 software (GraphPad Software, Inc., San Diego, CA).

\section{Results and discussion}

\subsection{Physicochemical characterization and stability}

The development of age appropriate paediatric formulations is paramount to enable children adherence to treatment. It encompasses multi-dimensional considerations including the administration route, the formulation technology, the dosage strength and other parameters as organoleptic properties, viscosity and $\mathrm{pH}$. For oral treatments, organoleptic characteristics are crucial for children compliance to therapeutic regimens. EMA paediatric investigation plan guidelines point out the particular relevance of organoleptic testing in the development of oral treatment for children (European Medicines Agency (EMA), 2006). The loss of pharmacological and sensory attributes depends on both the type of elaboration or storage process and the sensitivity of specific pharmaceutical components. Storage is accompanied by many changes, including chemical reaction and physical and structural changes which affect both pharmacological and sensory qualities. In paediatric solutions generally involves a series of interdependent phenomena. These processes affect, to a varying degree, the quality attributes of colour, texture and $\mathrm{pH}$.

Colour is an important attribute in pharmaceutical products, since it is perceived immediately by the consumer. It can also be a measurement of reactions extension in medicine since formed and/or degraded compounds may contribute to a specific colouration.

After preparation $(t=0)$ formulation 1 resulted to be a redispersible suspension, slightly pink with a characteristic fruity odour. There were no detectable changes in colour or odour in 
any sample during the two months of storage at the three controlled temperatures. On the other hand, formulation II (glucose free) resulted in a transparent solution also exhibiting light pink colour and fruity odour. When the vials were observed at naked eye after 7 days, it could be seen a white sediment non resoluble by shaking. This sediment was observed in samples stored at $4{ }^{\circ} \mathrm{C}$. This event was subsequently also corroborated by the SLD dose uniformity assay, from which, a noticeable reduction of the API in solution (formulation II) was detected at $t=7$ days.pH measurements showed values around 4.2 at $t_{0}$, these values remained almost constant over time, and thus no statistically significant differences were observed $(p>0.05)$ probably due to the presence of the citrate buffer that ensured a constant acid pH allowing SLD to keep solubilized in formulation II, because this $\mathrm{pH}$ value corresponds with the maximum solubility of SLD. The different solubility behaviour of SLD in those two simple syrups excipients utilized could be explained by the minor content of water in the syrup excipient with glucose compared to glucose free, what might difficult the API solubility in formulation I. Equally, the influence of the temperature on $\mathrm{pH}$ was studied by one-way ANOVA and no statistically significant differences were showed for any formulation $(p=0.7163$ and 0.8227 , formulation I and II respectively). However, despite significant differences were recorded as a function of time $(p<0.05)$, these were not considered relevant.

Particle size of the API in suspension can affect the uniformity of content since large particles will settle faster than their smaller counterparts (FDA, 2009). Equally, particle size distribution in the finished drug product dosage form is a critical parameter that significantly impacts the bioavailability and pharmacokinetics of the product (Kulshreshtha et al., 2010). Results of the particle size study of formulation I showed that size distribution at $t_{0}$ were $d_{10 \%}=3.24 \pm 0.2 \mu \mathrm{m}, d_{50 \%}=10.94 \pm 0.1 \mu \mathrm{m}$ and $d_{90 \%}=31.36 \pm 0.5 \mu \mathrm{m}$. A slight increase on day 90 compared to day 0 was observed in $d_{50 \%}$ and $d_{90 \%}$ with values of $12.86 \pm 0.7$ and $35.85 \pm 0.4 \mu \mathrm{m}$ respectively.

Oral viscosity plays an important role in the textural appreciation of liquid or fluid pharmaceutics. Oral assessments of viscosity correlate with both small-deformation and large-deformation rheological but there is still much speculation as to what forces actually operate in the mouth. Viscosity measurements have been performed with an imposed shear rate and the resulting shear stress was measured. With this type of test, it can be determined if the material is characterized by linear-viscous (Newtonian) behaviour (shear stress exponent $n=1$ ) in the investigated shear rate range or if it shows power-law behaviour $(n \neq 1)$. The potential dependence of the viscosity of the formulations on the shear rate is shown in Fig. 2. Formulation I graph shows constant viscosity values with increasing shear rate from 0 to $100 \mathrm{~s}^{-1}$ with constant slope that gives rise to a line, and thus Newtonian behaviour (Lee et al., 2009). Formulation II curve showed a small dependence on shear rate, showing a consistent, although relatively small, decrease in viscosity with increasing shear rate from 0 to $100 \mathrm{~s}^{-1}$. This is the typical behaviour of pseudoplastic fluids. In this case, the hysteresis loop is inappreciable, indicative that this solution did not show thyxotropy. It could be probably due to the presence of xanthan gum in formulation II (simple syrup glucose free excipient) the shearing action on the long chain molecule of xanthan gum. As the shearing stress is increased, the disarranged molecules start to align their long axes in the direction of the flow. This orientation reduces the resistance which further allows lowering of viscosity at increasing shearing stress (Martin, 1993). This result is in accordance with previous reports where it is mentioned that xanthan gum exhibits plastic or pseudoplastic flow (Ofner et al., 1996).

Viscosity values at $100 \mathrm{~s}^{-1}$ were $2.9 \pm 0.10 \mathrm{mPas}$ and $10.70 \pm 0.2 \mathrm{mPas}$ for formulation I and II respectively after preparation at $t_{0}$. The presence of xanthan gum in formulation II (simple syrup glucose free excipient) resulted in a substantial increase of the viscosity value with respect to Formulation I. No statistically significant differences were observed at the end of the storage period ( 90 days) at any temperature indicative of a desired rheological stability. This stability is an important requirement because the dosification process should ensure uniform distribution of the API in a suspension, especially among paediatric patients.

According to the obtained results of the method validation, the analytic method was lineal confirmed by the ANOVA of

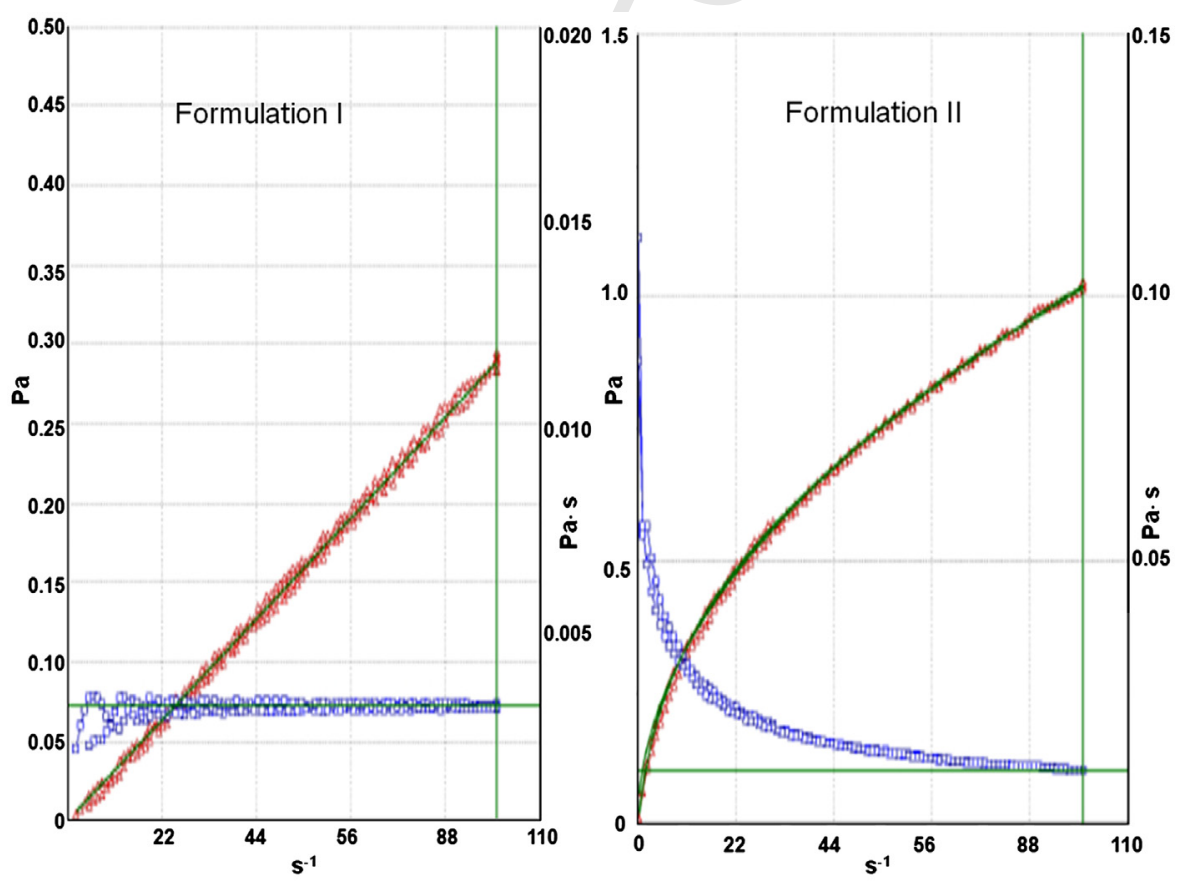

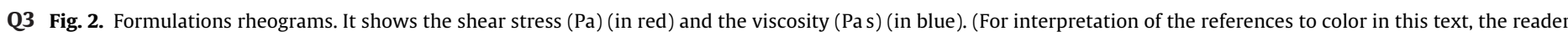
is referred to the web version of the article.) 


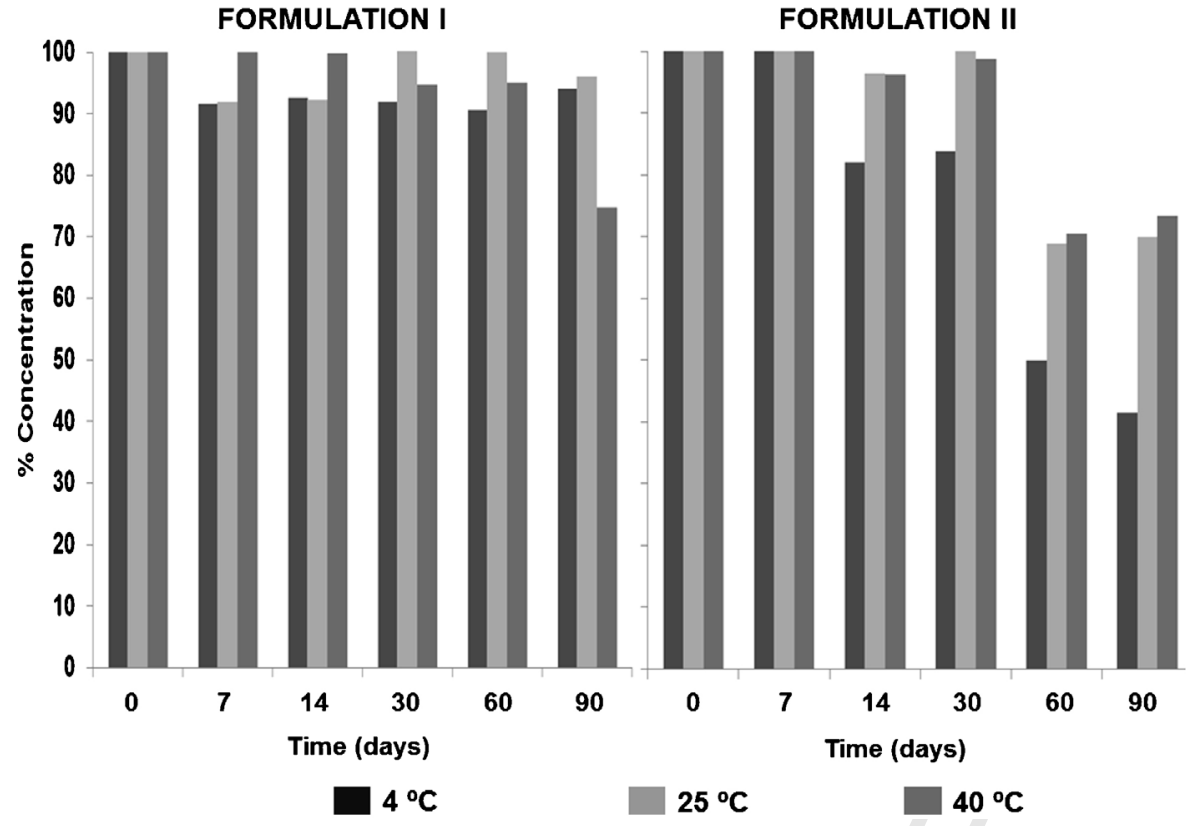

Fig. 3. Drug content (\%) in developed formulations over time at three temperatures.

the linear regression $(p>0.05)$ with determination coefficients $\left(r^{2}\right)>0.99$ in all curves. The LOD was $0.32 \pm 0.54 \mu \mathrm{g} / \mathrm{mL}$ and LOQ $0.97 \pm 0.11 \mu \mathrm{g} / \mathrm{mL}$. Accuracy (as relative error) as the percent deviation from the nominal concentration did no vary more than $10 \%$ from the expected. Precision in analysis in \% RSD values were less than $15 \%$.

Regarding uniformity of API content, as is shown in Fig. 3 the concentration of SLD in formulation I was maintained above $90 \%$ at 4 and $25^{\circ} \mathrm{C}$ for 90 days, however at $40^{\circ} \mathrm{C}$ this content was observed for 60 days. On the other hand, drug content in formulation II was maintained constant (above $90 \%$ ) at 25 and $40^{\circ} \mathrm{C}$ for 30 days, but at $4{ }^{\circ} \mathrm{C}$ this SLD content was achieved only for 7 days, at $t=15$ the drug concentration was $82.0 \pm 0.9 \%$, and $83.1 \pm 0.7 \%$ after 30 days. This decrease was confirmed by the presence of a white sediment making manifest the influence of low storage temperature in the solubility of the API.

\subsection{Optical stability assay}

Turbiscan ${ }^{\circledR}$ Lab is considered as a device which predicts the stability, being able to detect the formula destabilization before than the classical stability methods (microscopy, spectroscopy, turbidity and particle size analysis) easily up to 50 times earlier that the naked eye (Fernández Campos et al., 2012). Moreover, it provides real-time information on the destabilization process. When sedimentation process is produced, a backscattering increase versus time at the bottom of the sample is observed. When the sample suffers a creaming process, an increase of backscattering versus time on the top of the vial is observed. If the destabilization phenomenon occurs due to particle aggregation, a backscattering increase versus time can be observed over the whole height of the sample (Celia et al., 2009). If backscattering profiles have a deviation of $\leq \pm 2 \%$ it can be considered that there are no significant variations on particle size. Variations up to $\pm 10 \%$ indicate instable formulations.

Fig. 4 shows migration phenomena by local variations of the backscattering of formulation I corresponding to measurements on different hours. The left side of the curves corresponds to the bottom of the vial, whereas the right side corresponds to the top. As expected, it was an unstable system; it can be observed an increase of backscattering in the middle of the vial possibly due to the formation of aggregates by coalescence or flocculation. No flotation phenomenon was observed. These results were in accordance with those of particle size distribution, in which, at $t=90$ days an increase was recorded. Particles in suspension possess a surface free energy that makes the system unstable leading to particle settling. Free energy of the system depends on the total surface area and the interfacial tension between the liquid medium and the solid particles. Thus, in order to minimize this free energy, the system tends to decrease the surface area, which is achieved by formation of agglomerates (Kulshreshtha et al., 2010).

\subsection{Microbiological studies}

Microbial contamination in non sterile liquid formulations may cause foul odour, turbidity, and adversely affect to the palatability and appearance. Otherwise, high level of microorganisms may be hazardous to health especially in inmunocompromised patients.

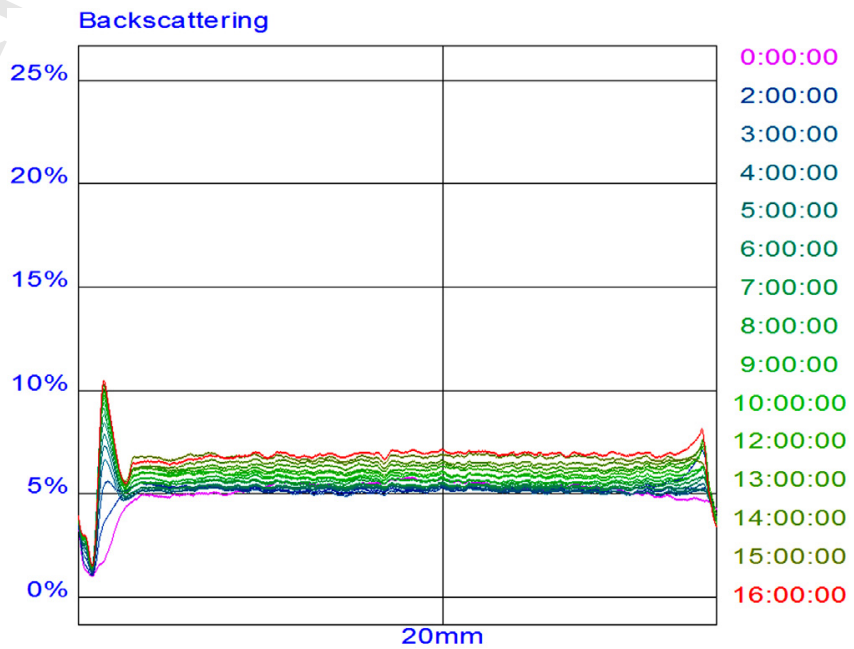

Fig. 4. Transmission profiles of formulation I. The left side of the curve corresponds to the bottom of the vial, whereas the right side corresponds to the sample behaviour on the top of the vial. 
For both formulations no E. coli contamination was observed and total bacteria count was less than $10^{2} \mathrm{cfu} / \mathrm{g}$ on day 90 of the study. Fungal contamination was also less than $10^{1} \mathrm{cfu} / \mathrm{g}$ in both. These results indicated that both formulations complied with the European Pharmacopoeia specifications on microbial examination of non-sterile product throughout 90 days.

\section{Conclusions}

Two new oral liquid formulations of SLD for paediatric use have been developed from pure powder, a suspension (formulation I) and a solution (formulation II), this latter suitable for diabetic patients. From obtained results it could be concluded that formulation I was stable from a physicochemical and microbiological point of view for 90 days at 4 and $25^{\circ} \mathrm{C}$, whereas at $40^{\circ} \mathrm{C}$ the API content remained constant in the acceptable limits for 60 days. On the other hand formulation II was stable for 30 days when was stored at 25 and $40^{\circ} \mathrm{C}$, however at $4{ }^{\circ} \mathrm{C}$ API concentrations under $90 \%$ were recorded at day 15 , this reduction was accompanied by the emergence of an non redispersible sediment, suggesting a decrease of SLD solubility at low temperature.

Obtained results also suggested that $\mathrm{pH}$ did not show statistically significant differences with the assayed conditions and the rheological behaviour also remained constant in both formulations. Finally formulations were microbiologically stable for at least 90 days. All these findings make possible to guarantee a correct dosification and administration of SLD when is formulated in liquid oral forms for the treatment of PPHN.

\section{Acknowledgment}

The authors would like to thank Acofarma S.A. for supplying sildenafil citrate and excipients.

\section{References}

Allen, L.V., 2008. Dosage form design and development. Clin. Ther. 30, $2102-2111$.

Archer, S.L., Michelakis, E.D., 2009. Phosphodiesterase type 5 inhibitors for pulmonary arterial hypertension. N. Engl. J. Med. 361, 1864-1871.

Atz, A.M., Wessel, D.L., 1999. Sildenafil ameliorates effects of inhaled nitric oxide withdrawal. Anesthesiology 91, 307-310.

Bauters, T., Claus, B., Willems, E., De Porre, J., Verlooy, J., Benoit, Y., Robays, H., 2012. What's in a drop? Optimizing strategies for administration of drugs in pediatrics. Int. J. Clin. Pharm. 4, 679-681.

Brion, F., Nunn, A.J., Rieutord, A., 2003. Extemporaneous (magistral) preparation of oral medicines for children in European hospitals. Acta Paediatr. 92, 486-490.

Celia, C., Trapasso, E., Cosco, D., Paolino, D., Fresta, M., 2009. Turbiscan lab expert analysis of the stability of ethosomes and ultradeformable liposomes containing a bilayer fluidizing agent. Colloids Surf. B: Biointerface. 72, 155-160.
Chua, S.S., Chua, H.M., Omar, A., 2010. Drug administration errors in paediatric wards: a direct observation approach. Eur. J. Pediatr. 169, 603-611.

Ernest, T.B., Elder, D.P., Martini, L.G., Roberts, M., Ford, J.L., 2007. Developing paediatric medicines: identifying the needs and recognizing the challenges. J. Pharm. Pharmacol. 59, 1043-1055.

European Medicines Agency(EMA), 2006. http://ec.europa.eu/health/files/eudralex/ vol-1/reg_2006_1901/reg_2006_1901_en.pdf (accessed 25.08.13.)

European Pharmacopoeia 7.0. http://www.edqm.eu/en/european-pharmacopoeiapublications-1401.html (accessed 25.08.13.).

FDA, 2009. http://www.fda.gov/iceci/inspections/inspectionguides/ucm074935 htm (accessed 25.08.13.).

Fernández Campos, F., Calpena Campmany, A.C., Rodríguez Delgado, G., López Serrano, O., Clares Naveros, B., 2012. Development and characterization of a nove nystatin-loaded nanoemulsion for the buccal treatment of candidosis: ultrastructural effects and release studies. J. Pharm. Sci. 101, 3739-3752.

Glass, B.D., Haywood, A., 2006. Stability considerations in liquid dosage forms extemporaneously prepared from commercially available products. J. Pharm. Pharm. Sci. 9, 398-426.

Gold, M.E., Wood, K.S., Byrns, R.E., Fukuto, J., Ignarro, L.J., 1990. NG-methyl-L-arginine causes endothelium-dependent contraction and inhibition of cyclic GMP formation in artery and vein. Proc. Natl. Acad. Sci. U. S. A. 87, 4430-4434

Hoehn, T., 2007. Therapy of pulmonary hypertension in neonates and infants. Pharmacol. Ther. 114, 318-326.

Humpl, T., Reyes, J.T., Holtby, H., Stephens, D., Adatia, I., 2005. Beneficial effect of oral sildenafil therapy on childhood pulmonary arterial hypertension: twelvemonth clinical trial of a single-drug, open-label, pilot study. Circulation 111, 3274-3280.

ICH, 2006. Validation of analytical procedures: text and methodology Q2 (R1), http://www.ema.europa.eu (accessed 28.08.13.).

Kulshreshtha, A.K., Singh, O.N., Wall, G.M., 2010. Pharmaceutical suspensions. In: From Formulation Development to Manufacturing. Springer, New York.

Lee, C.H., Moturi, V., Lee, E., 2009. Thixotropic property in pharmaceutical formulations. J. Control. Release 136, 88-98.

Martin, A., 1993. Physical Pharmacy. Lea \& Febiger, Philadelphia.

McEvoy, E., Donegan, S., Power, J., Altria, K., 2007. Optimisation and validation of a rapid and efficient microemulsion liquid chromatographic (MELC) method for the determination of paracetamol (acetaminophen) content in a suppository formulation. J. Pharm. Biomed. Anal. 44, 137-143.

Nahata, M.C., Allen, L.V., 2008. Extemporaneous drug formulations. Clin. Ther. 30, $2112-2119$.

Ofner, C.M., Schnaare, R.L., Schwartz, J.B., 1996. Reconstitutable oral suspensions. In: Lieberman, H.A., Rieger, M.M., Banker, G.S. (Eds.), Pharmaceutical Dosage Forms: Disperses Systems. Marcel Dekker, New York, pp. 247-249.

Santoveña, A., Hernández-Paiz, Z., Fariña, J.B., 2012. Design of a pediatric oral formulation with a low proportion of hydrochlorothiazide. Int. J. Pharm. 423, 360-364

Sola, A., Baquero, H., 2007. Oral sildenafil in neonatal medicine: tested in adults also used in neonatos. An. Pediatr. 66, 167-176.

Steinhorn, R.H., Kinsella, J.P., Pierce, C., Butrous, G., Dilleen, M., Oakes, M., Wessel D.L., 2009. Intravenous sildenafil in the treatment of neonates with persistent pulmonary hypertension. J. Pediatr. 155, 841-847.

Sundell-Bredenberg, S., Nyströn, C., 2001. The possibility of achieving an interactive mixture with high dose homogeneity containing an extremely low proportion of a micronized drug. Eur. J. Pharm. Sci. 12, 285-295.

The United States Pharmacopeial Convention, 2007. Compounded preparations. The United States Pharmacopeia 30-The Nacional Formulary 25, vol. 1. Port City Press, Baltimore, pp. 332.

Travadi, J.N., Patole, S.K., 2003. Phosphodiesterase inhibitors for persistent pulmonary hypertension of the newborn: a review. Pediatr. Pulmonol. 36, 529-535.

Wening, K., Breitkreutz, J., 2011. Oral drug delivery in personalized medicine: unme needs and novel approaches. Int. J. Pharm. 404, 1-9.

Wilson, D.G., MvArtney, R.G., Newcombe, R.G., McArtney, R.J., Gracie, J., Kirk, C.R. Stuart, A.G., 1998. Medication errors in paediatric practice: insights from a continuous quality improvement approach. Eur. J. Pediatr. 57, 769-774. 